\title{
Complete genome sequences of Clostridium perfringens Del1 strain isolated from chickens affected by necrotic enteritis
}

\author{
Charles Li ${ }^{1^{*}} \mathbb{D}$, Xianghe Yan² ${ }^{2^{*}}$ and Hyun S. Lillehoj ${ }^{1}$
}

\begin{abstract}
Background: Clostridium perfringens is ubiquitous in nature. It is a normal inhabitant in the intestinal tract of animals and humans. As the primary etiological agent of gas gangrene, necrosis and bacteremia, C. perfringens causes food poisoning, necrotic enteritis (NE), and even death. Epidemiology research has indicated that the increasing incidence of NE in poultry is associated with the withdrawal of in-feed antibiotic growth promoters in poultry production in response to government regulations. The recent omics studies have indicated that bacterial virulence is typically linked to highly efficient conjugative transfer of toxins, or plasmids carrying antibiotic-resistance traits. Currently, there is limited information on understanding of host-pathogen interaction in NE caused by virulent strains of C. perfringens. Elucidating such pathogenesis has practical impacts on fighting infectious diseases through adopting strategies of prophylactic or therapeutic interventions. In this report, we sequenced and analyzed the genome of $C$. perfringens Del1 strain using the hybrid of PacBio and Illumina sequencing technologies.

Results: Sequence analysis indicated that Del1 strain comprised a single circular chromosome with a complete 3,559,163 bp and 4 plasmids: pDel1_1 (82,596 bp), pDel1_2 (69,827 bp), pDel1_3 (49,582 bp), and pDel1_4 (49,728 bp). The genome had 3361 predicted coding DNA sequences, harbored numerous genes for pathogenesis and virulence factors, including 6 for antibiotic and antimicrobial resistance, and 3 phage-encoded genes. Phylogenetic analysis revealed that Del1 strain had similar genome and plasmid sequences to the CP4 strain.

Conclusion: Complete chromosomal and plasmid sequences of Del1 strain are presented in this report. Since Del1 was isolated from a field disease outbreak, this strain is a good source to identify virulent genes that cause many damaging effects of Clostridial infections in chicken gut. Genome sequencing of the chicken pathogenic isolates from commercial farms provides valuable insights into the molecular pathogenesis of C. perfringens as a gastrointestinal pathogen in food animals. The detailed information on gene sequencing of this important field strain will benefit the development of novel vaccines specific for C. perfringens-induced NE in chickens.
\end{abstract}

Keywords: Clostridium perfringens, CP Del1 strain, Complete genome sequence

\section{Background}

Clostridium perfringens $(\mathrm{CP})$ is a rod-shaped, spore-forming, Gram-positive anaerobic bacterium

\footnotetext{
*Correspondence: Charles.li@ars.usda.gov; Xianghe.yan@ars.usda.gov ${ }^{1}$ Animal Biosciences and Biotechnology Laboratory, Beltsville Agricultural Research Center, Agricultural Research Service-US Department of Agriculture, Beltsville, 10300 Baltimore Avenue, MD 20705, USA ${ }^{2}$ Environmental Microbial and Food Safety Laboratory, Beltsville Agricultural Research Center, Agricultural Research Service-US Department of Agriculture, Beltsville, 10300 Baltimore Avenue, MD 20705, USA
}

ubiquitous in nature and a large commensal bacterial population present in the intestines of humans and animals $[1,2]$. Annual economic losses to the world poultry industry associated with CP infections are estimated to be more than $\$ 6$ billion [3]. As the primary etiological agent of necrosis, bacteremia, and gas gangrene, $\mathrm{CP}$ causes food poisoning, necrotic enteritis (NE), chronic underperformance in commercial animal production, and even mortality $[4,5]$. Isolates of $\mathrm{CP}$ are commonly classified into Types A to E based on the 
toxins produced, and virulence is mainly attributed to their individual capacities to produce multiple toxins and extracellular enzymes $[1,6]$. Literature mining has been used to clearly demonstrate that horizontal gene transfer via plasmids and other extra-chromosomal elements can convert non-toxigenic $\mathrm{CP}$ strains into toxin producers $[7,8]$. In this study, the whole-genome sequencing of CP Del1 strain and comparative genomic analyses to published sequences are presented. Ultimately, genomic sequencing of different strains will enhance our understanding of the process by which commensal bacteria such as CP evolves as an intestinal pathogen in food animals. This will lead to the definition of putative vaccine targets to better protect chickens against $\mathrm{CP}$-induced $\mathrm{NE}$.

\section{Methods}

Bacterial strain, culture conditions and DNA isolation

A virulent CP Del1 strain (BioSample accession: SAMN06252079) was originally isolated from the intestines of a chicken on an NE-affected farm in Delaware, USA in 2009 and this strain was used to induce experimental NE in broiler chickens [3]. Bacteria were cultured anaerobically for $16 \mathrm{~h}$ and pellets were collected for DNA extraction using the cetyl trimethylammonium bromide method [9].

\section{Genomic DNA sequencing}

The complete genome sequence of CP Del1 was determined with the PacBio RS II platform (Pacific Biosciences, Menlo Park, CA, USA), performed and assembled at Genomic Resource Center, University of Maryland (Baltimore, MD, USA). A 20-kb DNA library was constructed using SMRTbell Template Preparation kit 1.0 according to the manufacturer's protocol and sequenced using single-molecule real-time (SMRT) sequencing technology with P6-C4 chemistry and 1 RS II SMRT cell, and the data were assembled de novo using the hierarchical genome assembly process (HGAP). Since PacBio sequencing has a higher singlepass error rate (15\%) than HiSeq platform does $(1.5 \%)$ [10], the assembled whole-genome shotgun sequences were verified with Illumina HiSeq 4000 (San Diego, CA, USA) performed by Novogene Inc. (Sacramento, CA, USA). Sequencing libraries were generated using NEBNex DNA Library Prep Reagent Set (New England Biolabs Inc, Ipswich, MA, USA) and index codes were added to each sample. The clustering of the indexcoded samples was performed on a cBot Cluster Generation System using HiSeq 3000/4000 PE Cluster Kit. After cluster generation, the libraries were sequenced on a HiSeq 4000 platform into paired-end 150-bp short reads.

\section{Assembly, genome annotation and genomic analysis}

Annotation was carried out by the NCBI Prokaryotic Genome Annotation Pipeline (released 2013). Information about this annotation pipeline can be found through the following web link: https://www.ncbi.nlm.nih.gov/ genome/annotation_prok.

\section{Quality assurance}

Genomic DNA was extracted from pure cultures of a single bacterial colony of Del1. The bacterium was identified as CP by biochemical identification kit and PCR product sequencing targeting $16 \mathrm{~S}$ rRNA and necrotic enteritis B-like toxin (netB) gene, as described previously [3]. Potential contamination of the genomic libraries by other microorganisms was evaluated using a BLAST search against the non-redundant database. A5-miseq includes a quality-checking step that detects putative mis-assemblies by identifying clusters of read pairs that map to disjointed locations in the assembled genome. This method did not detect any putative mis-assemblies. The internal sequencing errors resulting from Pacbio sequencing were corrected and verified by Illumina sequencing.

\section{Results and discussion}

\section{General genome characteristics}

The complete genome of CP Del1 was composed of a circular chromosome (Fig. 1a) and 4 plasmids (Fig. 1b). The genome had a chromosome of 3,559,163 bp long with a GC content of 28.3\%, 3556 genes (chromosome and plasmids), 3361 coding DNA sequences (CDSs) (chromosome), 30 rRNA genes (10 5S, 10 16S, and 10 23S), 94 tRNA genes and 4 non-coding RNAs (Table 1 ). The plasmids, designated as pDel1_p1, pDel1_p2, pDel1_p3 and pDel1_p4, were 82,596, 69,827, 49,582 and 49,728 bp long, respectively. The genomic annotation data for $\mathrm{CP}$ Del1 and 3 other CP reference strains, CP4, JP55 and ATCC13124, are summarized in Table 1. One striking difference was that CP4 had only 38 RNA genes while the 3 other CP strains contained 128 genes. This discrepancy may be accounted for by incomplete gene sequences of CP4 strain.

The Clusters of Orthologous Group (COG)-associated functional genes for CP Del1 and other bacterial starins are shown in Additional file 1: Table S1. Among the various COG categories in CP Del1, 6 comprised the largest proportions (each $\geq 5 \%$ of the total COG classifications) in order from largest to least: $\mathrm{R}$ [general function prediction only, 319 open reading frame (ORFs), 9.76\%], $\mathrm{S}$ (function unknown, 283 ORFs, 8.65\%), G (carbohydrate transport and metabolism, 203 ORFs, 6.21\%), K (transcription, 202 ORFs, 6.18\%), E (amino acid transport and metabolism, 181 ORFs, 5.54\%), and J (translation, ribosomal structure and biogenesis, 169 ORFs, 5.17\%). 

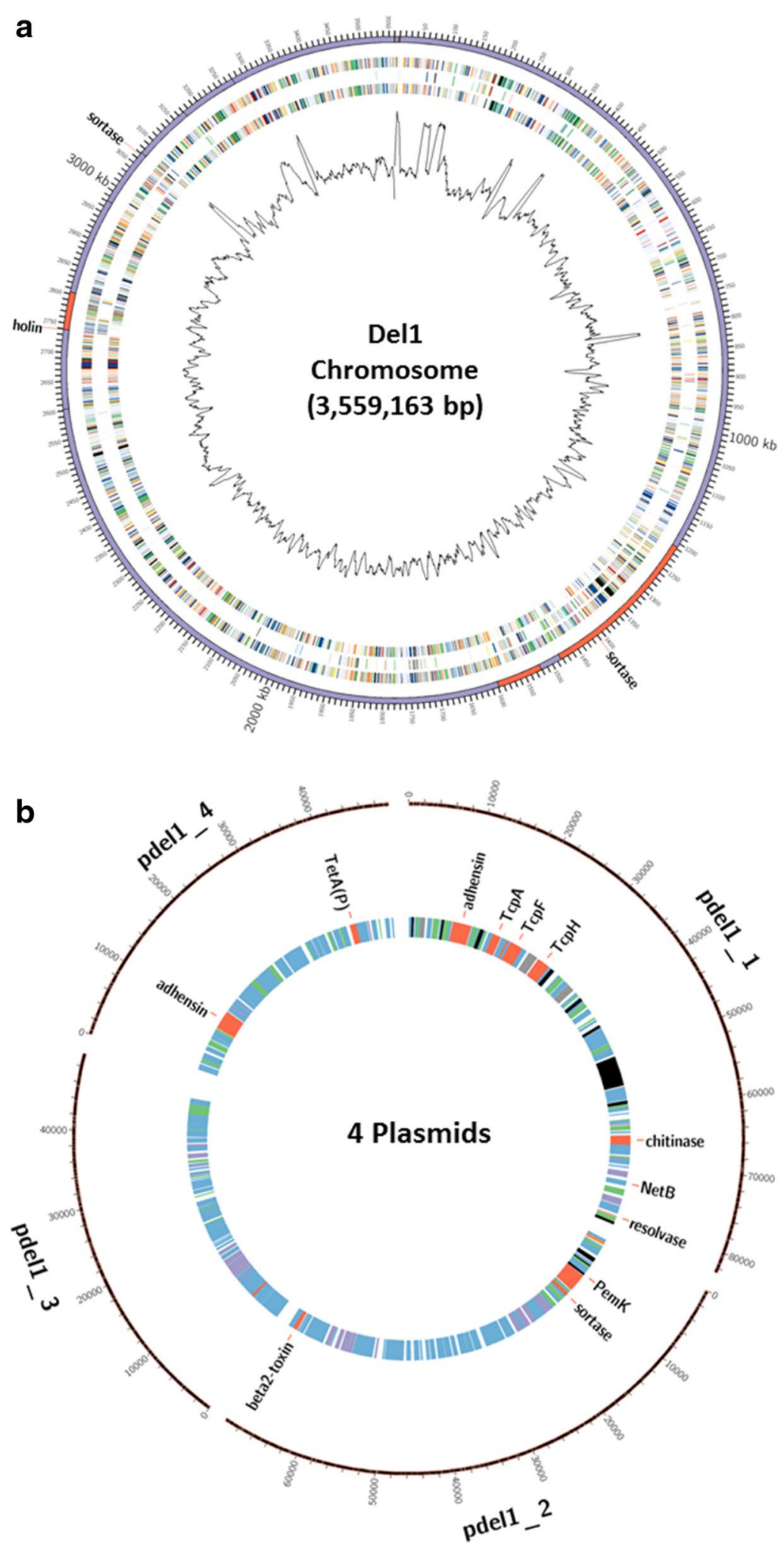

Fig. 1 Genomics characterization of C. perfringens (CP) strain Del1. a Genome comparison between CP Del1 and reference ATCC13124 strains; b 4 plasmids in Strain Del1 using CIRCOS. The cut off value of BLASTN homology was $90 \%$ in Fig. 1a. Outer ring: scale, 1st ring: all genes of Del1; 2 nd ring: genes less than 90\% homology to ATCC13124; 3rd ring: genes more than 90\% identity to ATCC13124; 4th ring: indicating the G + C content. Note: the size of Fig. 1a, b are not proportional to their actual nucleotide size 
Table 1 Summary in genome annotation data for Clostridium perfringens strain Del1 and 3 reference strains: CP4, JP55 and ATCC13124

\begin{tabular}{|c|c|c|c|c|}
\hline \multirow[t]{2}{*}{ Genome annotation } & \multicolumn{4}{|c|}{ Clostridium perfringens Strains } \\
\hline & DEL1 & $\mathrm{CP} 4$ & JP55 & ATCC13124 \\
\hline Genome accession\# & $\begin{array}{l}\text { NZ_CP019576.1 } \\
(3559163 \mathrm{bp})\end{array}$ & GCA_001414595.1 (latest) & $\begin{array}{l}\text { NZ_CP010993.1 } \\
\text { (3347300 bp) }\end{array}$ & NC_008261.1 (325666 bp) \\
\hline Sequence status & Complete genome & Contigs (98 contigs) & Complete genome & Complete genome \\
\hline Annotation date & $3 / 5 / 2017$ & $04 / 04 / 2017$ & $04 / 05 / 2017$ & $04 / 06 / 2017$ \\
\hline Assembly method & $\begin{array}{l}\text { Celera Assembler \& Genom- } \\
\text { ics Workbench v. V. 9.5.1 }\end{array}$ & Velvet v. 0.7.48 & $\begin{array}{l}\text { DNASTAR SeqMan v. } \\
\text { NGEN12 }\end{array}$ & na \\
\hline Annotation method & $\begin{array}{l}\text { Best-placed reference pro- } \\
\text { tein set; GeneMarks+ }\end{array}$ & $\begin{array}{l}\text { Best-placed reference pro- } \\
\text { tein set; GeneMarks+ }\end{array}$ & $\begin{array}{l}\text { Best-placed reference pro- } \\
\text { tein set; GeneMarks+ }\end{array}$ & $\begin{array}{l}\text { Best-placed reference protein } \\
\text { set; GeneMarks+ }\end{array}$ \\
\hline $\begin{array}{l}\text { Annotation software revi- } \\
\text { sion }\end{array}$ & 4.1 & 4.1 & 4.1 & 4.1 \\
\hline Genes (total) & 3556 & 3394 & 3287 & 2948 \\
\hline CDS (chromosome) & 3361 & 3293 & 3064 & 2801 \\
\hline Genes (RNA) & 128 & 38 & 128 & 128 \\
\hline rRNAs & $10,10,10(5 S, 165,235)$ & $1(5 S)$ & $10,10,10(5 S, 16 S, 23 S)$ & $8,8,8(5 S, 16 S, 23 S)$ \\
\hline tRNAs & 94 & 33 & 94 & 27 \\
\hline ncRNAs & 4 & 4 & 4 & 4 \\
\hline Pseudo genes (total) & 136 & 106 & 95 & 27 \\
\hline
\end{tabular}

Generally, all CP strains (Del1, JP55) exhibited comparable ranges in proportions of COG classifications.

\section{Comparative phylogenetic analysis and genomic variants among $C$. perfringens strains}

To date, 52 complete genome sequences of CP strains and their annotation data are available in the GenBank database. To compare these genomes, whole genome DNAsequence-based average nucleotide index (ANI) analysis was performed and phylogenetic trees were drawn based on genomic sequence analysis of these $52 \mathrm{CP}$ genomes (Fig. 2). The dendrogram illustrates that CP Del1 strain was the most closely related to $\mathrm{CP} 4$ strain, and clustered with JP55, MJR7757A and 5 other JFP strains. CP4 strain, whose genomic sequence has been characterized (9), is a pathogen that causes NE [11], and contains 3 highly conserved NE-associated loci that are designated NELoc-1 (42 kb), NELoc-2 (11.2 kb) and NELoc-3 (5.6 kb), with netB residing on an $\sim 42-\mathrm{kb}$ plasmid-encoded pathogenicity locus (NELoc-1) [12]. In CP Del1, netB gene resides in plasmid pDel1_1.

\section{Pathogenesis and virulence factors}

We also found numerous important genes involved in pathogenesis and virulence in the CP Del1 chromosome and plasmids (Additional file 1: Table S2). CP Del1 and CP4 strains share similar genes, with more genes absent in the strain ATCC13124 genome. Besides alpha-toxin phospholipase C (cpa plc), CP Dell genome also had genes encoding necrotic enteritis B-like toxin (netB), Beta 2 toxin (cpb2), mu-toxin (nagH-K), sialidase (nanH, nanI and nanJ), collagenase (peptidase_U32), and perfringoly$\sin \mathrm{O}$ (thera toxin, pfo). Alpha toxin (cpa-encoded) is the most toxic extracellular enzyme produced by $\mathrm{CP}$ type A. It is present in all types and essential for virulence, and its core protein, phospholipase $\mathrm{C}$, hydrolyzes both important constituents of eukaryotic cell membranes: phosphatidylcholine and sphingomyelin [13, 14]. NetB is a pore-forming toxin critical for induction of $\mathrm{NE}$ in chickens with predisposing factors, such as preceding coccidial infection and dietary manipulation [15, 16]. Sialidase cleaves terminal sialic acid residues in the alpha configuration linked to oligosaccharide chains present on proteins and lipids and functions as a nutritional factor to support bacterial growth and as a virulence factor during bacterial pathogenesis [1].

Studies on the genetic diversity of $C$. perfringens in mild NE-affected chickens indicated that more than $90 \%$ of all isolates from NE-specific organ lesions carried netB gene [17]. However, immunization with vaccines containing recombinant NetB toxin only partially protected progeny chickens from necrotic enteritis challenges [18]. Other virulence factors may additively contribute to the CP pathogenesis in NE induction. Global transcriptomic analysis performed on ligated intestinal loops in chickens following infection with a net ${ }^{+} \mathrm{CP}$ strain revealed that numerous virulence factors were significantly expressed in vivo, such as cpa, 


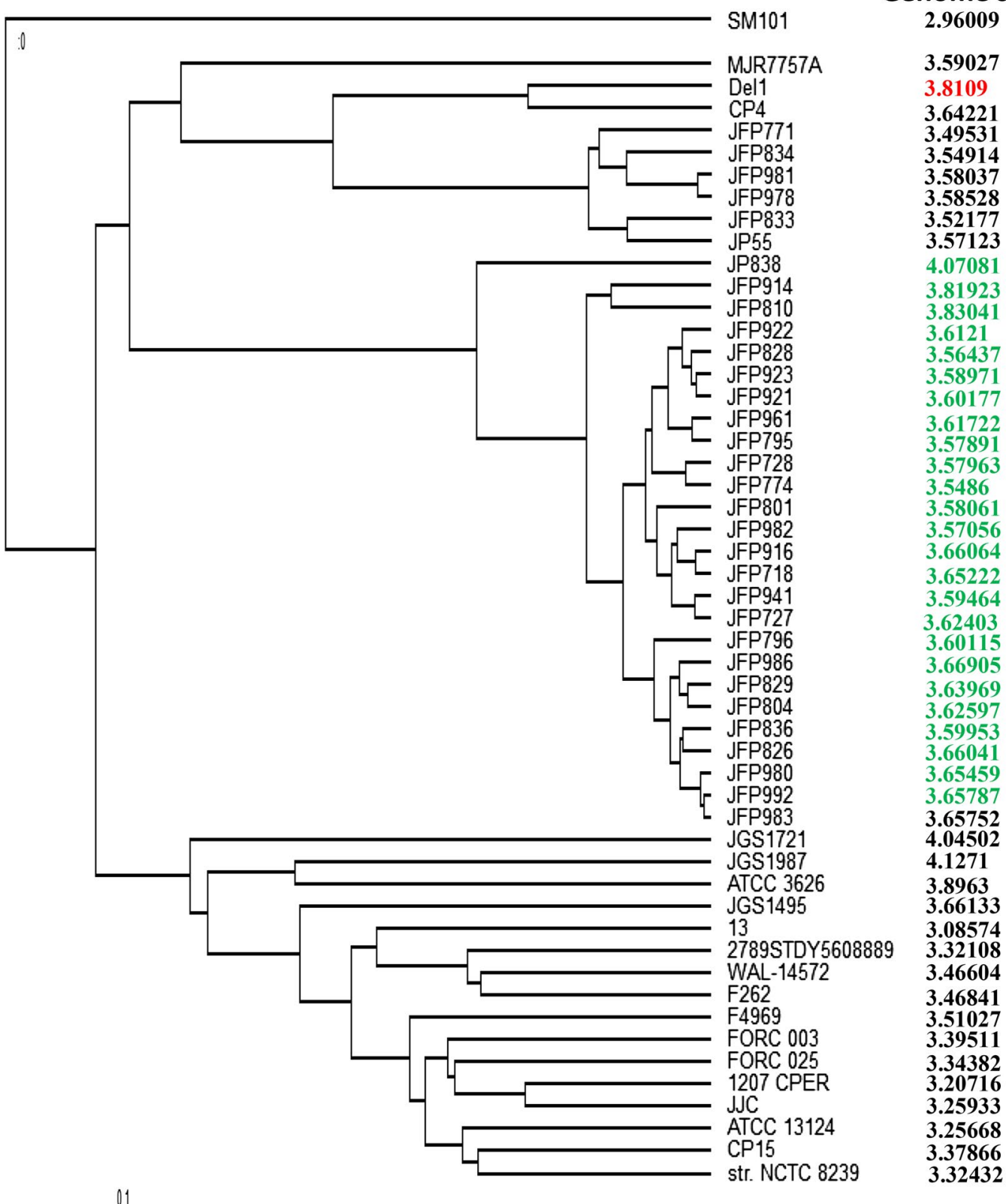

Fig. 2 Phylogenetic tree analysis of CP Del1 and other 51 published C. perfringens reference strains in genomic dendrogram by orthologous average nucleotide identity (ANI). The genome sizes are listed in the right

netB and sialidase genes [19]. Del1 strain is a virulent strain isolated from a field disease outbreak to be used as a challenge strain. Compared to CP4 strain, this Del1 strain contains some unique genes expressing adhensin, pilin of the toxin- coregulated-pilus (tcp cluster $\mathrm{C}-\mathrm{J})$, and programmed cell death toxin (PemK) which may be involved in multitude of pathogenesis functions, including adherence to eukaryotic cells, DNA uptake, and protein/toxin secretion, and therefore enhancing the pathogenesis and favoring the colonization and infection of C. perfringens as a gastrointestinal pathogen in food animals. Sequencing analysis also indicated that Del1 strain did not contain genes expressing Tpel toxin and surface protein CPE1231 as the CP4 strain had. Previously, it was reported that Tpel protein enhanced bacterial virulence and severity of NE [16]. 
Identifying bacterial important genes that encode toxin and virulence factors in genomics and transcriptomics approaches is critical for recombinant vaccine development, for example, cpa, netB, sialidase B, tpel, and PemK. In general, these genes contribute to toxin generation and bacterial colonization and growth. Strain Del1 is classified as Type A due to absence of beta, iota and epsilon toxins based on the definition of the presence of toxin genes in toxinotyping [1].

\section{Antibiotic-resistance genes}

By screening the genome and plasmid sequences against the GenBank library, we identified 6 antibiotic-resistance genes/clusters in CP Del1 genomes and plasmids. Specially these genes conferred resistance to tetracycline (total 3 genes: 1 gene encoding tetracycline ribosomal protection with Accession No: YP_001967743, 2 genes encoding tetracycline efflux pumps: BAB71966 and YP_001966009), 1 gene for class B beta-lactams (Subclass B1_136), as well as 1 gene for other antibiotics (YP_698526). Finally, another 1 gene encoded a major facilitator superfamily that was involved with antibiotic efflux pump (P96712), by which membrane transport proteins facilitated movement of small antibiotics across the cell membrane [20]. CP4 was also found to confer resistant to tetracycline [21]. Del1 strain was found in its susceptibility test to be resistant to tetracycline hydrochloride with minimal inhibitory concentrations of more than $256 \mu \mathrm{g} / \mathrm{mL}$.

\section{Identification of phage-encoded genes in Del1 strain}

Bacteriophage DNAs serve as mobile genetic elements which confer the antibiotic-resistance genes or virulence determinants by transduction, and ultimately contribute to genome diversification of bacterial hosts, with strain to strain variations in bacteriophage repertoire [22]. However, host specificity has routine been observed relative to the bacteriophages isolated from various CP strains. This is most likely due to evolution of the receptor in the bacterial hosts and anti-receptor molecules in bacteriophages (3). Using a previously described bioinformatics platform [23], we have determined specific genes in two regions (1224604-1235574 and 2737554-2740876) in Del1 genome which have high sequence similarities to Clostridium phage vB_CpeS-CP51, Clostridium phage PhiS63, and bacteriophage phi3626. Further, these regions also have low homology between them, indicating the prophages in Del1 strain are also strain specific. Sequence annotation predicts that majority of the genes in these two prophages-like areas encode hypothetical proteins.

\section{Future directions}

We have obtained a complete genome and 4 associated plasmid sequences of CP Del1 strain. The genomic analysis revealed that CP Del1 sequences consist of numerous genes encoding toxins and virulence factors, and include 6 that encode for proteins to confer antibiotic resistance, and 3 bacteriophage gene inserts. The improved understanding of the molecular pathogenesis of $\mathrm{CP}$-induced $\mathrm{NE}$ in chickens is necessary for development of effective treatments. Future studies should identify specific roles of pathogenesis/virulence factors, especially those present in the plasmids. Ultimately, we envision that this data and approach will guide us to the selection of strains and $\mathrm{CP}$ gene targets for further vaccine development.

\section{Additional file}

Additional file 1: Table S1. Numbers and proportions of general COGassociated functional genes for Clostridium perfringens strains Del1 and JP55. Table S2. The comparisons of virulence genes among Clostridium perfringens CP Del1, and 2 reference strains CP4 and ATCC13124.

\section{Abbreviations}

CP: Clostridium perfringens; NE: necrotic enteritis; netB: necrotic enteritis toxin B-like; CDS: coding DNA sequence; COG: clusters of orthologous group; ANI: average nucleotide identity.

\section{Authors' contributions}

$\mathrm{CL}, \mathrm{XY}$, and $\mathrm{HL}$ designed the study and contributed to the manuscript writing. $\mathrm{CL}$ also performed laboratory experiments, while XY conducted the data analysis. All authors read and approved the final manuscript.

\section{Acknowledgements \\ DNA Pacbio sequencing was completed using the genomic platform at Institute for Genome Sciences, University of Maryland at Baltimore, MD 21201. The DNA Illumina sequencing was performed by Novogene Inc, Sacramento, CA 95817. The authors also thank Dr. Ransom Baldwin and Dr. Xuechu Zhao for critical review of this manuscript. This work was supported by funding from ARS/USDA. \\ Mention of trade names or commercial products is solely for the purpose of providing specific information and does not imply recommendation or endorsement by the US Department of Agriculture.}

\section{Competing interests}

The authors declare that they have no competing interests.

\section{Availability of data and materials}

The data supporting the findings of this study are contained within the manuscript. The whole-genome and plasmid sequences have been deposited in the GenBank under the Accession Number CP019576 for bacterial Del1 chromosome, CP019577 for its plasmid1, CP019578 for plasmid 2, CP019579 for plasmid 3, and CP019580 for plasmid 4. The versions described in this report are the second version.

Ethics approval and consent to participate

No ethic issue was involved in this study.

\section{Funding}

This work was supported by Agricultural Research Service, US Department of Agriculture which had no role in study design, data collection and analysis, decision to publish, or preparation of the manuscript. 


\section{Publisher's Note}

Springer Nature remains neutral with regard to jurisdictional claims in published maps and institutional affiliations.

Received: 12 September 2017 Accepted: 13 November 2017

Published online: 21 November 2017

\section{References}

1. Li J, Adams V, Bannam TL, Miyamoto K, Garcia JP, Uzal FA, Rood Jl, McClane BA. Toxin plasmids of Clostridium perfringens. Microbiol Mol Biol Rev. 2013;77(2):208-33.

2. Brynestad S, Granum PE. Clostridium perfringens and foodborne infections. Int J Food Microbiol. 2002;74(3):195-202.

3. Li C, Lillehoj HS, Gadde UD, Ritter D, Oh S. Characterization of Clostridium perfringens strains isolated from healthy and necrotic enteritis-afflicted broiler chickens. Avian Dis. 2017;61(2):178-85.

4. Timbermont L, Haesebrouck F, Ducatelle R, Van Immerseel F. Necrotic enteritis in broilers: an updated review on the pathogenesis. Avian Pathol. 2011:40(4):341-7.

5. Zhou H, Lepp D, Pei Y, Liu M, Yin X, Ma R, Prescott JF, Gong J. Influence of pCP1NetB ancillary genes on the virulence of Clostridium perfringens poultry necrotic enteritis strain CP1. Gut Pathog. 2017;9:6.

6. Petit L, Gibert M, Popoff MR. Clostridium perfringens: toxinotype and genotype. Trends Microbiol. 1999;7(3):104-10.

7. Wisniewski JA, Traore DA, Bannam TL, Lyras D, Whisstock JC, Rood J. TcpM: a novel relaxase that mediates transfer of large conjugative plasmids from Clostridium perfringens. Mol Microbiol. 2016;99(5):884-96.

8. Gyles C, Boerlin P. Horizontally transferred genetic elements and their role in pathogenesis of bacterial disease. Vet Pathol. 2014;51(2):328-40.

9. Wilson K. Preparation of genomic DNA from bacteria. Curr Protoc Mol Biol. 2001;2:2-4

10. Schloss PD, Jenior ML, Koumpouras CC, Westcott SL, Highlander SK. Sequencing $16 \mathrm{~S}$ rRNA gene fragments using the PacBio SMRT DNA sequencing system. PeerJ. 2016;4:e1869.

11. Thompson DR, Parreira VR, Kulkarni RR, Prescott JF. Live attenuated vaccine-based control of necrotic enteritis of broiler chickens. Vet Microbiol. 2006:113(1-2):25-34
12. Lepp D, Roxas B, Parreira VR, Marri RR, Rosey EL, Gong J, Songer JG, Vedantam G, Prescott JF. Identification of novel pathogenicity loci in Clostridium perfringens strains that cause avian necrotic enteritis. PLoS ONE. 2010;5(5):e10795.

13. Awad MM, Bryant AE, Stevens DL, Rood JI. Virulence studies on chromosomal alpha-toxin and theta-toxin mutants constructed by allelic exchange provide genetic evidence for the essential role of alphatoxin in Clostridium perfringens-mediated gas gangrene. Mol Microbiol. 1995:15(2):191-202.

14. Rood JI. Virulence genes of Clostridium perfringens. Annu Rev Microbiol. 1998:52:333-60.

15. Keyburn AL, Bannam TL, Moore RJ, Rood Jl. NetB, a pore-forming toxin from necrotic enteritis strains of Clostridium perfringens. Toxins (Basel). 2010;2(7):1913-27.

16. Prescott JF, Smyth JA, Shojadoost B, Vince A. Experimental reproduction of necrotic enteritis in chickens: a review. Avian Pathol. 2016:45(3):317-22

17. Johansson A, Aspán A, Kaldhusdal M, Engström BE. Genetic diversity and prevalence of netB in Clostridium perfringens isolated from a broiler flock affected by mild necrotic enteritis. Vet Microbiol. 2010;144(1-2):87-92.

18. Keyburn AL, Portela RW, Ford ME, Bannam TL, Yan XX, Rood Jl, Moore RJ. Maternal immunization with vaccines containing recombinant NetB toxin partially protects progeny chickens from necrotic enteritis. Vet Res. 2013;44:108.

19. Parreira VR, Russell K, Athanasiadou S, Prescott JF. Comparative transcriptome analysis by RNAseq of necrotic enteritis Clostridium perfringens during in vivo colonization and in vitro conditions. BMC Microbiol. 2016;16(1):186

20. Fluman N, Bibi E. Bacterial multidrug transport through the lens of the major facilitator superfamily. Biochim Biophys Acta. 2009;1794(5):738-47.

21. Kulkarni RR, Parreira VR, Sharif S, Prescott JF. Clostridium perfringens antigens recognized by broiler chickens immune to necrotic enteritis. Clin Vaccine Immunol. 2006;13(12):1358-62.

22. Colomer-Lluch M, Imamovic L, Jofre J, Muniesa M. Bacteriophages carrying antibiotic resistance genes in fecal waste from cattle, pigs, and poultry. Antimicrob Agents Chemother. 2011:55(10):4908-11.

23. Li PE, Lo CC, Anderson JJ, Davenport KW, Bishop-Lilly KA, Xu Y, Ahmed S, Feng S, Mokash VP, Chain PS. Enabling the democratization of the genomics revolution with a fully integrated web-based bioinformatics platform. Nucleic Acids Res. 2017;45(1):67-80.

\section{Submit your next manuscript to BioMed Central and we will help you at every step:}

- We accept pre-submission inquiries

- Our selector tool helps you to find the most relevant journal

- We provide round the clock customer support

- Convenient online submission

- Thorough peer review

- Inclusion in PubMed and all major indexing services

- Maximum visibility for your research

Submit your manuscript at www.biomedcentral com/submit
BioMed Central 